\title{
The Association between Frailty and Cognition in Elderly Women
}

\author{
Ji-Young Kang, Cheol-Hwan Kim*, Eun-Ju Sung, Ho-Cheol Shin, Woon-Jung Shin, Keun-Hyeong Jung \\ Department of the Family Medicine, Kangbuk Samsung Hospital, Sungkyunkwan University School of Medicine, Seoul, Korea
}

Background: Frailty refers to the loss of physiologic complexity and the associated decline in ability to withstand stressors as one gets older. It is defined as unintentional weight loss, exhaustion, weakness, slow walking speed, and low physical activity. According to several western studies, frailty is associated with cognitive impairment, but there have been few studies about the relationship between frailty and cognitive impairment in Korea. Thus, the purpose of this study is to analyze the relationship between cognition and factors related to frailty such as grip strength, walking speed, physical activity, and depression, among female patients older than 65 in Korea.

Methods: A total of 121 subjects among the outpatients of the department of family medicine at Kangbuk Samsung Hospital who did not meet the exclusion criteria were included in this study. We divided the participants into 2 groups, according to the Korean version of the Montreal Cognitive Assessment (MoCA) score: 1 group with subjects that had normal cognition and the other group with patients that had impaired cognition. A comparison was made between the 2 groups in regards to the factors related to frailty, and we completed equation that predicting cognition from the frailty related factors.

Results: Compared with the impaired cognition group, the subjects in the normal cognition group had higher hand strength, and walked faster $(\mathrm{P}<0.001)$. There was no statistically significant difference in physical activity between the 2 groups $(\mathrm{P}=0.19)$. When multiple linear regression analysis was performed using age, grip strength, and walking speed as the predictor variables and MoCA score as the dependent variable, the regression coefficients were calculated to be: $-0.2015,0.2294,1.2372$, and -0.1436 , respectively $(\mathrm{P}<0.05)$.

Conclusion: In Korean female patients who are older than 65 years of age, cognition tends to decline as grip strength decreases, walking speed gets slower, depression becomes more severe, and as age increases.

Keywords: Frail; Cognition; Hand Strengths; Walking; Depression 


\section{INTRODUCTION}

Frailty means that the ability of a person to cope with stress is reduced and it leads to negative outcomes such as falls, hospitalizations, disability, and deaths. ${ }^{1)}$ Frailty ultimately results in dependence and disabilities that are associated with the reduced physiological function. ${ }^{1)}$ Frailty is an important consideration for the elderly, because it is important to maintain a life of independence and a guaranteed quality of life for them. Although the definition of frailty is not yet clear, ${ }^{2)}$ Fried et al. ${ }^{1)}$ proposed 5 objective components of the criteria including: unintentional weight loss, exhaustion, muscle weakness, slowness while walking, and low levels of activity. A case was defined as frailty if it corresponded with at least 3 of the 5 components of the criteria. Rockwood and Mitnitski ${ }^{3}$ defined frailty as the accumulation of mental and physical defects.

Cognitive impairment refers to decreased intellectual function, which ranges widely from forgetfulness to dementia. ${ }^{4)}$ Mild cognitive impairment is mild cognitive decline that is not associated with significant functional disability, ${ }^{5)}$ and it increases the probability of suffering from dementia. ${ }^{4)}$ In dementia, there is severe cognitive decline accompanied by decreased physical function, which ultimately results in the affected elderly being completely physically dependent on others. ${ }^{4}$

Recent studies have identified the association between frailty and cognition. In a study by Avila-Funes et al., ${ }^{6}$ study participants were divided into 2 groups according to the Fried criteria: the 'frail' and 'robust' groups. It was found that the prevalence of cognitive impairment is higher in the frail group (22\%) compared with the robust group (10\%). A similar finding was reported by Yassuda et al., ${ }^{7}$ where the prevalence of cognitive impairment was higher in the frail group (39\%) compared with the robust group (16\%). Samper-Ternent et al. ${ }^{8)}$ investigated a study group that consisted of the elderly who had a mini-mental state examination score $>21$, and the study participants were divided into frail and robust groups. When the study participants were followed up after 10 years, the degree of cognitive decline was greater in patients of the frail group compared with those in the robust group. ${ }^{8)}$
Shim et al. ${ }^{9)}$ divided their study population of the elderly into frail and robust groups according to the study of osteoporotic fractures (SOF) frailty index, and when the patients were followed up after 1 year, the risk of cognitive decline was higher for patients in the 'frail' group compared with those in the 'robust' group (odds ratio, 3.57).

In this way, Western studies suggest that there is a correlation between physical frailty and cognition, but there are few studies investigating this relationship in Korea. Because a series of processes that lead to frailty as aging is thought to affect the cognitive decline associated with brain aging, understanding the correlation between physical frailty and cognition may provide insights on how to prevent and manage frailty and cognitive decline. Therefore, the purpose of this study is to analyze the correlation between the factors associated with physical frailty and cognition in the Korean female elderly. This study was conducted at our hospital due to the relatively large proportion of female patients in our family medicine department.

\section{METHODS}

\section{Study Population}

Female patients aged 65 years or older who visited the department of family medicine at Gangbuk Samsung Hospital between 11 January 2013 and 14 August 2014 were considered for participation in the study. Among these patients, those who underwent the assessment of physical frailty and cognition were assessed for eligibility ( $n=153)$. Patients were excluded from the study in the following cases: the patients did not agree to undergo the study assessments ( $n=13)$; the results of the assessments were not appropriate $(n=15)$; and the presence of an underlying disease such as Parkinson's disease or stroke that deemed the patient unsuitable for the study $(n=4)$. One hundred and twentyone subjects were enrolled into the final study participant group. Figure 1 illustrates the study subject selection process.

\section{Demographic Characteristics and Health Status}

We investigated the demographics and the clinical characteristics of

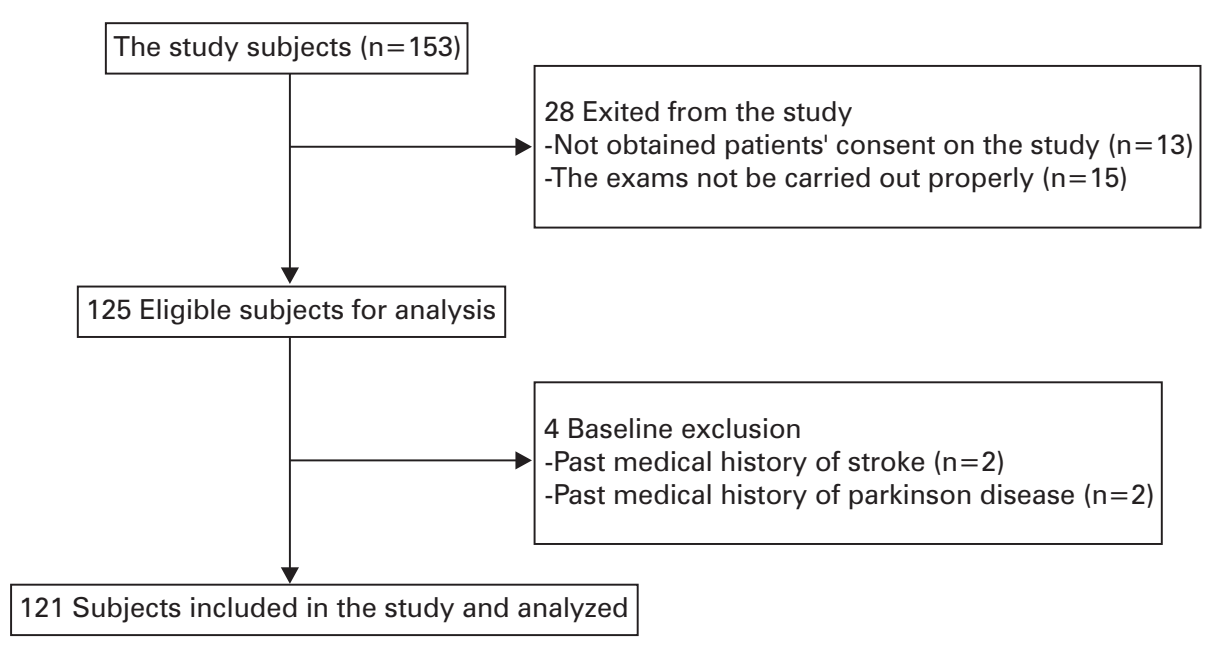

Figure 1. The study subject selection process. 
the study subjects. The investigation was carried out by using self-assessment questionnaires. The demographic information included age, education level, and economic status, while the clinical characteristics included falls, unintentional weight loss, morbidity, and the number of current ongoing medication. Information about education level was also reviewed and categorized into 3 groups: education level lower than elementary school, educated to the middle/high school level, and university educated. Economic status was assessed by monthly household income which was divided into the following categories: $<100$ won, $101-300$ won, 301-500 won, $>501$ won, and unknown. Falls was defined as the occurrence of falls more than 2 times during the past 6 months. Geriatric depression scale is a self-assessment questionnaire consisting of 30 questions that are answered with 'yes' or 'no.'10) The patients are considered to be suffering from mild depression or depression if the score is $>15 .{ }^{11)}$ Weight loss was defined as unintentional weight loss of more than $5 \mathrm{~kg}$ in the past 6 months. We investigated chronic diseases such as hypertension, diabetes mellitus, osteoarthritis, osteoporosis, stroke, coronary artery disease, heart failure, arrhythmia, cancer, chronic obstructive pulmonary disease, dementia, and Parkinson's disease.

\section{The Assessment of Physical Frailty}

There are a number of indicators of physical frailty. ${ }^{12)}$ Among these, well-established indicators are walking speed, ${ }^{13,14)}$ physical activity, ${ }^{15,16)}$ weight loss, ${ }^{17,18)}$ hand grip strength, ${ }^{19,20)}$ and sense of balance. ${ }^{21)}$ The Cardiovascular Health Study frailty index developed by Fried et al. ${ }^{1)}$ encompasses the following factors: weight loss, exhaustion, muscle weakness, slowness while walking, and low levels of activity. Another frailty index developed by Rockwood et al. ${ }^{22,23)}$ takes into account: cognition, emotional state, motivation, communication, motor ability, incontinence, the activities of daily living, nutrition, social support, and morbidity. There is a frailty index developed for the Korean people which encompasses 8 factors including: overall health status, medications, nutritional status (body weight), emotional state (depression), incontinence, walking ability, and communication. ${ }^{24)}$ Consulting these references, we adopted 3 factors related to physical frailty out of the 5 factors specified in the Fried frailty criteria. The 3 chosen factors were walking speed, hand grip strength (to assess muscle weakness), and physical activity, because they can be measured objectively by assessors. We excluded the weight loss and exhaustion factors as the result for the former relied on patients' memory, while the latter is subjective.

Muscle strength was evaluated through right hand grip strength by using Lavisen electronic hand grip dynamometer KS 301 (Lavisen Co. Ltd., Namyangju, Korea). Assessors instructed subjects to grip the dynamometer by positioning the medial phalange of the third finger perpendicular to the handle, spreading both arms straight toward the floor whilst keeping both arms away from the body, and then grabbing the handle with maximum force. The unit of hand grip strength was kilograms. Walking speed was evaluated by assessors inside the outpatients' exam room. Subjects were instructed to walk a straight distance of 4 meters at usual walking speed along a green straight line on the floor. Walking speed was recorded using a stop watch. Using the short physical performance battery (SPPB) protocol, points were awarded in the following manner: 4 points if the time taken was $<4.82$ seconds, 3 points if time taken was $>4.82$ seconds but $<6.20$ seconds, 2 points if the time taken was $>6.21$ seconds but $<8.70$ seconds, and 1 point if the time taken $>8.70$ seconds. To evaluate physical activity, the subjects responded to the question of "How many times do you exercise or are doing physical activity with moderate intensity per week?", by selecting their answer from 5 categories that ranged from 'none' to 'almost every day'

\section{Assessment of Cognition}

Cognition was evaluated by using the Korean version of the Montreal Cognitive Assessment (MoCA-K). MoCA-K is a tool developed to screen mild cognitive impairment. ${ }^{25)}$ It consists of a short-term memory test (5 points), space-time configuration function tests including a clock drawing test (3 points) and a 3-dimensional block drawing test (1 point), a trail-making test-B ( 1 point) to test for task performance, a language proficiency test (1 point), a 2 -item verbal abstraction task (2 points), a concentration test (1 point), a number subtraction test ( 3 points), forward and backward digit span ( 2 points), and a time and space orientation test ( 6 points), animal naming test ( 3 points), repeating after complex sentences test ( 2 points). The total possible score is 30 points. The cutoff score was 22 for screening mild cognitive impairment or dementia. ${ }^{26)}$

\section{Statistical Analysis}

We divided study subjects into 2 groups according to MoCA-K score, 1 group with normal cognition and the other with impaired cognition. The 2 groups were then compared in regards to their demographics and the proportion of patients with factors related to frailty, including: right hand grip strength, walking speed, physical activity, and depression. Analysis was performed by using Fisher's exact test and Pearson's chi-square test. Multivariate linear regression analysis was performed, using age, hand grip strength, walking speed, and geriatric depression scale score as predictive variables, and MoCA-K score as the dependent variable. These analyses were performed to demonstrate how MoCA-K scores vary depending on the values measured for each factor related to frailty. Statistical analysis was performed by using PASW SPSS ver. 18.0 (SPSS Inc., Chicago, IL, USA), and statistical significance was defined by $\mathrm{P}<0.05$.

\section{RESULTS}

The study subjects' demographic and clinical characteristics are presented in Table 1 . The age of the impaired cognitive group was older, and education level was lower, than the normal cognitive group $(\mathrm{P}=0.004, \mathrm{P}<0.001)$. The impaired cognitive group had more experiences of falls than the normal cognitive group $(\mathrm{P}=0.048)$. However, there was no significant difference between the two group regarding weight loss, body mass index (BMI), smoking history, family monthly income, the 
Table 1. Baseline characteristics of the study subjects according to cognitive function $(n=121)$

\begin{tabular}{|c|c|c|c|}
\hline \multirow{2}{*}{ Characteristic } & \multicolumn{2}{|c|}{$\begin{array}{c}\text { Cognitive function by Montreal } \\
\text { Cognitive Assessment-Korean version }\end{array}$} & \multirow[t]{2}{*}{ P-value } \\
\hline & Normal $(n=60)$ & Low $(n=61)$ & \\
\hline Age (y) & & & 0.004 \\
\hline 65-69 & $26(43.3)$ & $13(21.3)$ & \\
\hline $70-74$ & $20(33.3)$ & $15(24.6)$ & \\
\hline $75-80$ & $11(33.3)$ & $22(36.1)$ & \\
\hline$\geq 81$ & $3(5.0)$ & $11(18.0)$ & \\
\hline Body mass index ${ }^{\dagger}$ & & & 0.84 \\
\hline$\leq 18.4$ & $2(3.3)$ & $2(3.3)$ & \\
\hline $18.5-23$ & $10(16.7)$ & $14(23.0)$ & \\
\hline $23.1-24.9$ & $37(61.7)$ & $33(54.1)$ & \\
\hline$\geq 25.0$ & $11(18.3)$ & $12(19.7)$ & \\
\hline Education level & & & $<0.001$ \\
\hline None/elementary & 19 (31.6) & $37(60.6)$ & \\
\hline Middle/high & $34(56.7)$ & $23(37.7)$ & \\
\hline University & $7(11.7)$ & $1(1.6)$ & \\
\hline Family monthly income (won) & & & 0.26 \\
\hline$\leq 1,000,000$ & $15(25.0)$ & $13(21.3)$ & \\
\hline $1,010,000-3,000,000$ & $16(26.7)$ & $10(16.4)$ & \\
\hline $3,010,000-5,000,000$ & 7 (11.7) & $6(9.8)$ & \\
\hline$\geq 5,010,000$ & $6(10.0)$ & $4(6.6)$ & \\
\hline Not mentioned & $16(26.7)$ & $28(45.9)$ & \\
\hline No. of chronic disease ${ }^{\ddagger}$ & & & 0.83 \\
\hline 0 & $9(15.0)$ & $6(9.8)$ & \\
\hline 1 & $16(26.7)$ & $19(31.1)$ & \\
\hline 2 & $16(26.7)$ & $15(24.6)$ & \\
\hline 3 & $12(20.0)$ & $11(18.0)$ & \\
\hline$\geq 4$ & $7(11.7)$ & $10(16.4)$ & \\
\hline No. of current medication $\$$ & & & 0.87 \\
\hline 0 & $5(8.3)$ & 7 (11.5) & \\
\hline 1 & $25(41.7)$ & $20(32.8)$ & \\
\hline 2 & $15(25.0)$ & 18 (29.5) & \\
\hline 3 & $11(18.3)$ & $12(19.7)$ & \\
\hline$\geq 4$ & $4(6.7)$ & $4(6.6)$ & \\
\hline Smoking & & & 1.00 \\
\hline Current & $2(3.3)$ & $2(3.3)$ & \\
\hline Past & $1(1.7)$ & $2(3.3)$ & \\
\hline Never & $57(95.0)$ & $57(93.4)$ & \\
\hline Weight loss" & & & 0.11 \\
\hline Yes & $5(8.3)$ & $11(18.0)$ & \\
\hline No & 55 (91.7) & $50(82.0)$ & \\
\hline Falls in previous 6 months & & & 0.04 \\
\hline Yes & $18(30.0)$ & $29(47.5)$ & \\
\hline No & $42(70.0)$ & $32(52.5)$ & \\
\hline
\end{tabular}

Values are presented as number (\%), unless otherwise indicated.

*From Pearson chi-square test or Fisher's exact test. ${ }^{\dagger}$ Calculated as weight in kilograms divided by height in meters. ${ }^{*}$ Hypertension, diabetes mellitus, osteoarthritis, osteoporosis, coronary artery disease, heart failure, arrhythmia, chronic obstructive pulmonary disease, cancer, Alzheimer disease, thyroid disease. ${ }^{\S}$ Antihypertensives, diabetes mellitus medications, tranquilizers, antidepressants, dementia medications, analgesics, antihistamines, vasodilators, vasoconstrictors, bisphosphonates, selective estrogen receptor modulators, opioids, herb medications. "Defined as at least $5 \mathrm{~kg}$ loss in previous 6 months.

number of chronic diseases, and the number of medication taken by the study participants.

Table 2 presents the differences between the 2 groups in regards to
Table 2. Factors related to frailty of the study subjects according to cognitive function (MoCA-K)

\begin{tabular}{|c|c|c|c|}
\hline Variable & $\begin{array}{l}\text { MoCA-K } \geq 23 \\
\quad(n=60)\end{array}$ & $\begin{array}{c}\text { MoCA-K } \leq 22 \\
\quad(n=61)\end{array}$ & P-value* \\
\hline Right grip strength (kg) & & & $<0.001$ \\
\hline$\leq 14.5$ & 8 (13.3) & $33(54.1)$ & \\
\hline $14.6-18.7$ & $24(40.0)$ & 16 (26.2) & \\
\hline$\geq 18.8$ & $28(46.7)$ & $12(19.7)$ & \\
\hline Walking speed ${ }^{\dagger}$ (4 m) & & & $<0.001$ \\
\hline$>8.70 \mathrm{~s}$ & $2(3.3)$ & $10(16.4)$ & \\
\hline $6.21-8.70 \mathrm{~s}$ & $4(6.7)$ & $18(29.5)$ & \\
\hline $4.82-6.20 \mathrm{~s}$ & 19 (31.7) & 17 (27.9) & \\
\hline$<4.82 \mathrm{~s}$ & 35 (58.3) & $16(26.2)$ & \\
\hline Physical activity ${ }^{\ddagger}$ (no./wk) & & & 0.19 \\
\hline None & $24(40.0)$ & $32(52.5)$ & \\
\hline$<1 / w k$ & $6(10.0)$ & $5(8.2)$ & \\
\hline $1-3 /$ wk & $17(28.3)$ & $7(11.5)$ & \\
\hline $4-5 /$ wk & $4(6.7)$ & $5(8.2)$ & \\
\hline$>5 / w k$ & $9(15.0)$ & $12(19.7)$ & \\
\hline $\begin{array}{l}\text { Depression according to } \\
\text { geriatric depression scale }\end{array}$ & & & 0.001 \\
\hline$\geq 16$ & $28(46.7)$ & $47(77.0)$ & \\
\hline$\leq 15$ & $32(53.3)$ & $14(23.0)$ & \\
\hline
\end{tabular}

Values are presented as number (\%), unless otherwise indicated.

MoCA-K, Montreal Cognitive Assessment-Korean version.

*From Pearson chi-square test or Fisher's exact test. 'Estimated by short physical performance battery protocol. "Defined as exercising until one gets sweaty.

Table 3. Multivariate linear regression to assess how MoCA-K scores vary with the level of the factors related to frailty

\begin{tabular}{lcc}
\hline \multicolumn{1}{c}{ Predictors } & Regression coefficient (MoCA-K) & P-value $^{*}$ \\
\hline Constant & 30.707 & $<0.001$ \\
Age $(\mathrm{y})$ & -0.2015 & 0.005 \\
Geriatric depression scale & -0.1436 & 0.01 \\
Right grip strength $(\mathrm{kg})$ & 0.2294 & 0.003 \\
Walking speed $^{\dagger}(4 \mathrm{~m})$ & 1.2372 & 0.007 \\
\hline
\end{tabular}

MoCA-K, Montreal Cognitive Assessment-Korean version.

${ }^{*}$ From Pearson chi-square test. 'Walking velocity was put in by points estimated by short physical performance battery protocol.

factors related to frailty, including: hand grip strength, walking speed, physical activity, and the prevalence of depression. The normal cognitive group had higher hand grip strength and faster walking speeds than the impaired cognitive group $(\mathrm{P}<0.001)$. There was no significant difference between the 2 groups in regards to physical activity $(\mathrm{P}=0.19)$. The prevalence of depression, as evaluated by the geriatric depression scale, was higher in the impaired cognitive group than the normal cognitive group ( $77 \%$ vs. $46.7 \%, \mathrm{P}=0.001$ ).

Table 3 presents the results of the multivariate linear regression analysis where age, hand grip strength, walking speed, SPPB protocol score, and geriatric depression scale score were used as predictive variables, and MoCA-K score was used as the dependent variable. The regression coefficients of age, geriatric depression scale score, hand grip strength, and walking speed were $-0.2015,-0.1436,0.2294$, and 1.2372 , respectively. Thus, the degree of cognitive function can be cal- 
culated by using the equation below, which was made by using multivariate linear regression.

Cognitive function (in females aged $\geq 65$ years) $=30.707-0.2015 \times$ age$0.1436 \times$ (geriatric depression scale score $)+0.2294 \times($ right grip strength)+1.2372×(walking speed)

If the MoCA-K score which is being used as the dependent variable is below 22, the subject is suspected to suffer from cognitive impairment.

\section{DISCUSSION}

The results of our study shows that cognition tends to decline in Korean female subjects older than 65 years. As their age increases, hand grip strength becomes weaker, walking speed gets slower, and depressive moods are more severe. These results are similar to those reported in previous studies. Auyeung et al. ${ }^{27)}$ reported that physical frailty is associated with cognitive impairment in older subjects after 4-year follow-up. In that study, the factors related to physical frailty (body weight, hand grip strength, and the speed to rise from a chair) were significantly associated with cognition in males $(\mathrm{P}<0.01)$, while for females, hand grip strength was correlated with cognition $\left.(\mathrm{P}<0.05) .{ }^{27}\right)$ Lojko et al. ${ }^{28)}$ reported that highly active senile people had better working memory, time-space cognition, and attention comparing to groups of medium and low physical activity $(\mathrm{P}<0.05)$. In comparison to the results reported from these studies, our results show that weakening of hand grip strength and slower walking speed were associated with cognitive decline, while physical activity was not related to cognition. In Korea, Shim et al. ${ }^{9)}$ divided senile subjects into frail and robust groups according to the SOF frailty index, and when subjects were followed up after 1 year, it was found that the degree of the cognitive decline was larger in the frailty group compared with the robust group. Their study used the SOF frailty index which is based on the following 3 factors related to frailty: unintentional weight loss, the inability to rise from a chair 5 times without using the arms, and reduced energy level. In contrast to this study, we evaluated physical frailty on the basis of the Fried frailty index, and focused on examining whether each factor related to frailty correlates with cognition.

There are various mechanisms that explain the link between physical frailty and cognition. First, neuropathological mechanisms of Alzheimer's disease such as neurofibrillary tangles and amyloid plaque lead to cognitive impairment and also affect physical frailty. Buchman et al. ${ }^{29)}$ found that physical frailty worsens with increasing severity of Alzheimer's disease pathology. In the analysis of the correlation between factors related to physical frailty and Alzheimer's disease pathology, weakened hand grip strength, decreased BMI, and slower walking speed were related to the severity of Alzheimer's disease pathology. The second mechanism is that depression mediates the relationship between physical frailty and cognition. Mezuk et al. ${ }^{30)}$ found a positive correlation between frailty and depression. Moreover, results from the meta-analysis conducted by Lee et al. ${ }^{31)}$ showed that depressive patients had decreased function in psychomotor activity, visual learning, memory, and execution compared to healthy people. Like the aforementioned studies, the results of our study shows that depression was more prevalent in the impaired cognitive group, and cognitive function declines with higher geriatric depression scale scores. Based on the results of these studies, we can presume that depression mediates the correlation between frailty and cognition. The third mechanism is inflammatory reactions. Stress reactions increase proinflammatory cytokines ${ }^{32)}$ and diseases that induce the inflammatory reaction increases levels of interleukin-1 and tumor necrosis factor. The ageing process increases interleukin-6, and these cytokines promote catabolic reactions which decreases lean body mass and muscle, leading to frailty. ${ }^{33,34)}$ In addition, peripheral pro-inflammatory cytokines increase levels of central pro-inflammatory cytokines that generate neurotoxicity, leading to cognitive impairment. ${ }^{35-37)}$ Hence, we can presume that inflammatory reactions induce physical frailty and cognitive impairment by reducing muscle and increasing levels of central cytokines, respectively. Fourth, there is a nutritional mechanism to explain the relationship between physical frailty and cognition. If senile people do not have adequate intake of nutrition, this results in decreases in fat and muscle levels, leading to physical frailty. ${ }^{1)}$ Nourhashemi et al. ${ }^{38)}$ showed that in elderly women, a group of subjects with sarcopenia had an increased risk of cognitive impairment compared with a group that had normal muscle mass, and a group with lower fat composition had increased risk of cognitive impairment compared with a group that had higher fat composition. Thus, if one's body fat and muscles decreases owing to deficiency in nutritional intake, it can increase the risk of physical frailty and cognitive impairment.

The results of our study show that out of the factors related to frailty, age, hand grip strength, walking speed, and depression are associated with cognition. These results can be explained by the aforementioned mechanisms, where neuropathology that damages cognitive function induces physical frailty, depression and inflammatory reactions mediate the association with frailty, and nutritional deficiency cause decrease in fat and muscle levels which lead to frailty and cognitive impairment.

One of the limitations of this study is that we cannot demonstrate causality in the association between physical frailty and cognition because it is a cross-sectional study. However, on the basis of results from previous studies that showed physical frailty affects cognition, we suggest that physical frailty and depression can be risk factors for cognition. Another limitation of the study is the relatively small study population, and only female patients were included as study subjects. However, by restricting the study population to females, we can specifically collect results for the target population of this study. A third limitation is that the study population consisted of relatively healthy outpatients who could independently complete physical activity and the physical performance exam. However, the purpose of our study is to understand the relationship between physical frailty and impaired cognition in order to find out the new methods preventing these 2 conditions. 
Hence, it will not significantly affect the results of our study. Finally, by adopting only 3 factors (walking speed, hand grip strength, and physical activity) out of the 5 factors specified by the Fried frailty criteria, this is also a limitation.

The significance of our study is that it demonstrates the correlation between each factor related to frailty and cognition, for the first time in Korea.

Weakened hand grip strength and slowness while walking were among the factors related to physical frailty that were associated with cognition. To find conclusive evidence to support these results, future studies need to compare the degree of physical frailty to the degree of cognitive decline after follow-up of the subjects in a longitudinal study.

\section{CONFLICT OF INTEREST}

No potential conflict of interest relevant to this article was reported.

\section{REFERENCES}

1. Fried LP, Tangen CM, Walston J, Newman AB, Hirsch C, Gottdiener J, et al. Frailty in older adults: evidence for a phenotype. J Gerontol A Biol Sci Med Sci 2001;56:M146-56.

2. Ma SH, Jeung KY, Hong SH, Shim EY, Yoo SH, Kim MY, et al. Correlation between frailty level and disability of the elderly and frailty related factors. Korean J Fam Med 2009;30:588-97.

3. Rockwood K, Mitnitski A. Frailty in relation to the accumulation of deficits. J Gerontol A Biol Sci Med Sci 2007;62:722-7.

4. Robertson DA, Savva GM, Kenny RA. Frailty and cognitive impairment: a review of the evidence and causal mechanisms. Ageing Res Rev 2013;12:840-51.

5. Allegri RF, Glaser FB, Taragano FE, Buschke H. Mild cognitive impairment: believe it or not? Int Rev Psychiatry 2008;20:357-63.

6. Avila-Funes JA, Amieva H, Barberger-Gateau P, Le Goff M, Raoux N, Ritchie K, et al. Cognitive impairment improves the predictive validity of the phenotype of frailty for adverse health outcomes: the three-city study. J Am Geriatr Soc 2009;57:453-61.

7. Yassuda MS, Lopes A, Cachioni M, Falcao DV, Batistoni SS, Guimaraes VV, et al. Frailty criteria and cognitive performance are related: data from the FIBRA study in Ermelino Matarazzo, São Paulo, Brazil. J Nutr Health Aging 2012;16:55-61.

8. Samper-Ternent R, Al Snih S, Raji MA, Markides KS, Ottenbacher KJ. Relationship between frailty and cognitive decline in older Mexican Americans. J Am Geriatr Soc 2008;56:1845-52.

9. Shim EY, Ma SH, Hong SH, Lee YS, Paik WY, Seo DS, et al. Correlation between frailty level and adverse health-related outcomes of community-dwelling elderly, one year retrospective study. Korean J Fam Med 2011;32:249-56.

10. Yesavage JA, Brink TL, Rose TL, Lum O, Huang V, Adey M, et al. Development and validation of a geriatric depression screening scale: a preliminary report. J Psychiatr Res 1982-1983;17:37-49.

11. Kim JY, Park JH, Lee JJ, Huh Y, Lee SB, Han SK, et al. Standardization of the Korean version of the geriatric depression scale: reliability, validity, and factor structure. Psychiatry Investig 2008;5:232-8.
12. Gobbens RJ, van Assen MA. The prediction of ADL and IADL disability using six physical indicators of frailty: a longitudinal study in the Netherlands. Curr Gerontol Geriatr Res 2014;2014:358137.

13. Gill TM, Allore H, Holford TR, Guo Z. The development of insidious disability in activities of daily living among community-living older persons. Am J Med 2004;117:484-91.

14. Rosano C, Newman AB, Katz R, Hirsch CH, Kuller LH. Association between lower digit symbol substitution test score and slower gait and greater risk of mortality and of developing incident disability in wellfunctioning older adults. J Am Geriatr Soc 2008;56:1618-25.

15. Van Den Brink CL, Picavet H, Van Den Bos GA, Giampaoli S, Nissinen A, Kromhout D. Duration and intensity of physical activity and disability among European elderly men. Disabil Rehabil 2005;27:341-7.

16. Landi F, Onder G, Carpenter I, Cesari M, Soldato M, Bernabei R. Physical activity prevented functional decline among frail community-living elderly subjects in an international observational study. J Clin Epidemiol 2007;60:518-24.

17. Arnold AM, Newman AB, Cushman M, Ding J, Kritchevsky S. Body weight dynamics and their association with physical function and mortality in older adults: the Cardiovascular Health Study. J Gerontol A Biol Sci Med Sci 2010;65:63-70.

18. Al Snih S, Raji MA, Markides KS, Ottenbacher KJ, Goodwin JS. Weight change and lower body disability in older Mexican Americans. J Am Geriatr Soc 2005;53:1730-7.

19. Gill TM, Murphy TE, Barry LC, Allore HG. Risk factors for disability subtypes in older persons. J Am Geriatr Soc 2009;57:1850-5.

20. Giampaoli S, Ferrucci L, Cecchi F, Lo Noce C, Poce A, Dima F, et al. Hand-grip strength predicts incident disability in non-disabled older men. Age Ageing 1999;28:283-8.

21. Onder G, Penninx BW, Ferrucci L, Fried LP, Guralnik JM, Pahor M. Measures of physical performance and risk for progressive and catastrophic disability: results from the Women's Health and Aging Study. J Gerontol A Biol Sci Med Sci 2005;60:74-9.

22. Rockwood K, Song X, MacKnight C, Bergman H, Hogan DB, McDowell I, et al. A global clinical measure of fitness and frailty in elderly people. CMAJ 2005;173:489-95.

23. Rockwood K, Stadnyk K, MacKnight C, McDowell I, Hebert R, Hogan DB. A brief clinical instrument to classify frailty in elderly people. Lancet 1999;353:205-6.

24. Hwang HS, Kwon IS, Park BJ, Cho B, Yoon JL, Won CW. The validity and reliability of Korean frailty index. J Korean Geriatr Soc 2010;14:191-202.

25. Nasreddine ZS, Phillips NA, Bedirian V, Charbonneau S, Whitehead V, Collin I, et al. The Montreal Cognitive Assessment, MoCA: a brief screening tool for mild cognitive impairment. J Am Geriatr Soc 2005; 53:695-9.

26. Lee JY, Lee DW, Cho SJ, Na DL, Jeon HJ, Kim SK, et al. Brief screening for mild cognitive impairment in elderly outpatient clinic: validation of the Korean version of the Montreal Cognitive Assessment. J Geriatr Psychiatry Neurol 2008;21:104-10.

27. Auyeung TW, Lee JS, Kwok T, Woo J. Physical frailty predicts future cognitive decline: a four-year prospective study in 2737 cognitively normal older adults. J Nutr Health Aging 2011;15:690-4.

28. Lojko D, Palys W, Czajkowska A, Wieczorowska-Tobis K, Lukasik S, Gorna K, et al. Association of cognitive performance with the physical activity and body mass index in middle-aged and older rural inhabit- 
ants. Eur Rev Med Pharmacol Sci 2014;18:3645-52.

29. Buchman AS, Schneider JA, Leurgans S, Bennett DA. Physical frailty in older persons is associated with Alzheimer disease pathology. Neurology 2008;71:499-504.

30. Mezuk B, Edwards L, Lohman M, Choi M, Lapane K. Depression and frailty in later life: a synthetic review. Int J Geriatr Psychiatry 2012;27: 879-92.

31. Lee RS, Hermens DF, Porter MA, Redoblado-Hodge MA. A meta-analysis of cognitive deficits in first-episode Major Depressive Disorder. J Affect Disord 2012;140:113-24.

32. Rosano C, Marsland AL, Gianaros PJ. Maintaining brain health by monitoring inflammatory processes: a mechanism to promote successful aging. Aging Dis 2012;3:16-33.

33. Roubenoff R, Roubenoff RA, Cannon JG, Kehayias JJ, Zhuang H, Dawson-Hughes B, et al. Rheumatoid cachexia: cytokine-driven hypermetabolism accompanying reduced body cell mass in chronic inflamma- tion. J Clin Invest 1994;93:2379-86.

34. Ershler WB, Keller ET. Age-associated increased interleukin-6 gene expression, late-life diseases, and frailty. Annu Rev Med 2000;51:24570.

35. Banks WA, Farr SA, Morley JE. Entry of blood-borne cytokines into the central nervous system: effects on cognitive processes. Neuroimmunomodulation 2002-2003;10:319-27.

36. Stamatovic SM, Keep RF, Andjelkovic AV. Brain endothelial cell-cell junctions: how to "open" the blood brain barrier. Curr Neuropharmacol 2008;6:179-92.

37. Perry VH. Contribution of systemic inflammation to chronic neurodegeneration. Acta Neuropathol 2010;120:277-86.

38. Nourhashemi F, Andrieu S, Gillette-Guyonnet S, Reynish E, Albarede $\mathrm{JL}$, Grandjean $\mathrm{H}$, et al. Is there a relationship between fat-free soft tissue mass and low cognitive function?: results from a study of 7,105 women. J Am Geriatr Soc 2002;50:1796-801. 